\title{
Multiple stressors, state-dependence and predation risk - foraging trade-offs: toward a modern concept of trait-mediated indirect effects in communities and ecosystems.
}

\author{
Oswald J Schmitz ${ }^{1}$ and Geoffrey C Trussell ${ }^{2}$
}

\author{
Addresses \\ ${ }^{1}$ School of Forestry and Environmental Studies, Yale University, 370 Prospect Street, \\ New Haven, CT 06515 \\ ${ }^{2}$ Marine Science Center, Northeastern University, East Point, Nahant, MA 01908
}

Corresponding Author: Oswald Schmitz. School of Forestry and Environmental Studies, Yale University, 370 Prospect Street, New Haven, CT 06515; Oswald.schmitz@yale.edu 
Trait-mediated indirect effects driven by organismal foraging-predation risk trade-offs can determine food web structure and ecosystem functioning. How this trade-off is shaped by organismal state in relation to multiple environmental stressors remains poorly understood. Attention to this issue is fundamentally important in an era of global change where multiple stressors increasingly present unique challenges for populations of organisms. The challenges are compounded by geographic patterns in adaptation that influence organismal state and the scope of trade-off responses. We argue for a modern approach that links evolutionary history to state-dependence to determine the scope of organismal foraging-predation risk trade-off behavior in the face of multiple stressors. Such integration will enhance predictions of the ecological consequences of organism living in different environmental contexts.

\section{Introduction}

A defining moment in linking the study of animal behavior with the study of food chain interactions within ecosystems arguably began with a publication by Sih [1], followed four years later by a paper by Abrams [2]. The two papers triggered a fundamental change in how ecologists came to understand species interactions within food chains. They introduced the idea that predators can control food chain structure and dynamics by inducing anti-predator behavior responses in their prey. The papers advanced a general principle that in order to maximize their fitness prey species in food chains effectively must balance a trade-off between consuming their resources against becoming resources for their predators. This disarmingly simple principle created the potential to explain spatial and temporal context dependency in nature and strength of food chain interactions [3-5]. It further opened the possibility to predict emergent context dependency in the level of ecosystem functioning [3, 6, 7].

Early work held that the trade-off was reconciled either by prey species becoming vigilant, thereby shifting their foraging time budget; or becoming evasive, thereby shifting between foraging habitats and refuge habitats. Both forms of prey response reduced their impact on resources, often with important consequences for the species composition and dynamics of communities. Hence, the effects of predators on communities were mediated by shifts in prey behavioral traits. Such processes, along with others driven by predator-induced changes in prey morphological traits, became known broadly as trait-mediated indirect effects [3]. Developing the concept of trait-mediated indirect effects set the stage to advance a long sought $[8,9]$ operational way to connect evolutionary ecology with community and ecosystem functioning.

Advancing a modern concept of trait-mediated indirect effects means further recognizing that populations of species are ensembles of individuals that display considerable within and among population variation in traits $\left[10^{*}\right]$. Therefore, enhancing understanding of the food chain and ecosystem level implications of behavior requires adopting a relational approach [7*] that connects food chain interactions or ecosystem functioning to variation in trait expression among individuals in different environmental contexts (Figure 1). Variation in phenotypic trait expression, in turn, is dependent on the internal state of individuals, which is determined by individual morphology, physiology, or energetic and nutrient status [10-13, 14*-16]. Such reductionism offers an expanded, generalizable perspective because it can fundamentally connect 
how phenotypic state links to the nature of a species' trophic interactions within different environmental contexts (Figure 1).

Environmental context can be defined as the combination of environmental stressors (e.g., temperature, disturbance, etc.) that organisms face within a particular habitat [17]. Predators - or rather the risk they impose - can then be considered one among many sources of environmental stress [6]. The behavioral trade-off challenge facing organisms then becomes one of responding to multiple environmental stressors in an effort to gain nutrients to maximize fitness $[15,18]$. The capacity of organisms to behaviorally respond to multiple stressors is ultimately shaped by the degree of phenotypic variation in their physiological or morphological traits.

Here we argue that advancing a trait-based approach is key to developing a deeper understanding of context dependency in community and ecosystem functioning. Such an approach links phenotypic variation within populations to state-dependence in organismal responses to multiple stressors. This approach embraces the growing recognition that within organismal state is often central to both motivating or constraining how prey species trade-off foraging and avoiding predation in the face of multiple environmental stressors $[13,14,19 * 20 *-22 *]$. Indeed, such an approach even helps to explain the emergence of spatial patterning in the nature and strength of species interactions [22].

We advance the case however, that there is a need to consider organismal state-dependence in two important ways (Figure 1). The first way — within population state-dependence - is widely considered in conventional state-dependent analyses of behavioral plasticity $[11,18]$. Here, individuals are assumed to exhibit adaptive behavioral or physiological responses to the ecological conditions they face during the course of their life-cycles. For example individuals in low food environments (or in poor body condition) may be motivated to trade-off foraging against avoiding predation risk differently than individuals in high food environments (or in good body condition). Individuals in poor condition may tolerate greater predation risk because the risk of starvation outweighs that of predation. Alternatively, individuals in good condition may enhance their risk avoidance [13] because they can ride out pulses of risk or are engaging in asset protection $[13,23,24]$. The second, less appreciated, way-among-population statedependence - emerges from the evolutionary history of individuals within local populations. Local adaptation in individual physiology or morphology may be shaped by selection that varies across different environments. Such adaptation can create potential constraints on an individual's ability to exhibit foraging-predation risk trade-off plasticity [24]. As an example, early climate change work assumed that tropical species would fare well under climate change scenarios because they are accustomed to coping with thermal stress. But, temperate species, which face widely varying thermal regimes during the course of their lifetime, may fare equally well and in some circumstances better with environmental warming because of countergradient variation $[25,26]$. The degree of variation expressed among populations across geographic space may vary along a continuum. This continuum could range from individuals within populations being entirely phenotypically plastic to individuals within populations having plasticity constrained completely by purely genetic adaptation (e.g., genetic polymorphism or genetic canalization) to local habitat contexts (Figure 1). Selection can operate across this continuum whenever plasticity has an adaptive genetic basis. We contend therefore that analyzing state-dependence through the lens of both ecological context and evolutionary history 
will lead to a fully integrative approach to eco-evolutionary interactions by recognizing that local population structure could shape the evolution of trait plasticity and vice-versa [27-29*].

\section{State-dependence: blending analyses of plasticity and local adaptation}

Classic analyses of trait-mediated interactions have largely assumed that prey trade-off responses represent adaptive phenotypically plastic adjustment to predation risk [7]. Insight about plasticity (e.g., its mean and variance within and among populations) has then largely come from experiments in which prey individuals with different trait magnitudes (e.g. body size) are drawn from single populations and then experimentally exposed either to predator risk cues or to cuefree conditions. Experiments examining additional stressors like toxins, pollutants or rising temperature, layer on these stressors by factorially combining them with levels of predator cues [e.g. 30-34].

However, the expanded eco-evolutionary approach we advocate here evaluates potential for local adaptation, including local adaptation in the nature and strength of phenotypically plastic responses. This calls for blending the use of factorial designs with the use of transplant experiments across environmental gradients [20, 29, 35, 36]. Such designs draw individuals from populations in different environmental contexts and measure their performance in transplant as well as native sites [e.g., 20,36]. The power of this approach is that it considers the potential for adaptive variation across the environmental landscape to shape how individuals respond to stressors such as predation risk. Moreover, geographic patterns in adaptation (i.e., cogradient versus countergradient) to one stressor may enhance or constrain individual capacity to respond to another stressor. This is fundamentally important because stressors (e.g., temperature and predation risk or nutrient loading and predation risk) may synergistically or antagonistically perturb the same physiological pathways in individuals with important consequences for prey state that ultimately shapes prey decision-making and hence the nature of their trophic interactions $[20,37]$.

As a case in point, concerted effort $[19,36]$ to examine interactions between climate warming (both projected and natural) scenarios and predation on prey responses revealed contrasting outcomes that can be best explained by geographic differentiation in population adaptation to warming. In the Gulf of Maine, predation risk from predatory green crabs (Carcinus maenas) influences snail (Nucella lapillus) behavior and physiological performance on rocky shore communities: snails reduce their foraging rates and retreat to refuge habitats when green crab predation risk is present. Snails from a population at the warmer, southern limit of their range that were simultaneously exposed to projected climate warming scenarios and green crab predation risk in the lab exhibited no growth, indicating that these combined stressors placed snails at their energetic edge [36]. But, experiments replicated across a latitudinal temperature gradient found that individual snails originating from a cooler, northern population and reared at the southern, warmer end of the geographic range outperformed those that remained at their native site when facing predation risk. Hence, the positive effects of elevated temperature on foraging and growth (countergradient variation) offset the negative effects of predation risk, [sensu 24]. These contrasting patterns likely emerge because the two snail populations have evolved different thermal performance curves in response to geographic variation in thermal 
regimes that set the stage for context-dependency in how organisms respond to other stressors. Enhanced warming likely placed southern snails on a suboptimal part of their thermal performance curve limiting their capacity to also tolerate the additional stress of predation risk whereas for northern snails warming moved them to a more favorable part of their thermal performance curve, offsetting the negative effects of predation risk.

Such context dependency can also arise purely from adaptive phenotypically plastic responses to differences in rearing conditions of organisms during their life-cycle development. The context dependency can change organismal state, which in turn can have lasting impacts on organismal responses to stressors including consequences for organismal stoichiometry [33, 34].

\section{Stressors, organismal performance and implications for stoichiometry}

Stress from predation risk can change organismal stoichiometry, (i.e., the internal body carbon:nitrogen:phosphorus $[\mathrm{C}: \mathrm{N}: \mathrm{P}]$ balance) through shifts in digestive and metabolic processes that accommodate shifting nutrient demands of stressed individuals [6, 18, 38-43]. Theory predicts that prey exposed to chronic predation risk should increase their metabolic rates as an adaptive response that puts organisms in a heightened state of alertness and agility [6, 39]. Stressed prey should then shift their nutrient intake and consume more carbohydrate-C. This prediction derives from the idea that stressed animals should switch their life history strategies from one devoted to $\mathrm{N}$-demanding growth or reproduction to one of fulfilling $\mathrm{C}$-demanding maintenance functions to cope with the added stress $[38,39]$. Some experiments have shown that such shifts in nutrient intake can indeed occur between conditions with and without perceived predation risk $[38,39]$. Others, however, have shown that stressed organisms increase consumption of protein-N [40-43]. These differences may be explained by organismal state dictated by life-history or dietary adaptations. In some cases, prey such as frog tadpoles or juvenile fish avoid predation by accelerating growth and development of morphology that enhances predator escape performance, which comes at the expense of growth and development for reproductive performance [40-43]. In other cases, dietary specialization consigns prey to increase resource consumption (because they cannot switch diets) to increase the efficiency of

protein-N extraction. Doing this enables organisms to maximize growth rate to reach a predationsize refuge. However, this again comes at the expense of growth and development for reproduction [38].

These responses may, however, be idiosyncratic to experimental rearing conditions (e.g. temperature or nutrient supply). Changing the rearing conditions could conflate or confound these responses $[20,21,32 *-34 *]$, especially if there is variation in local population capacities to exhibit phenotypic plasticity. This again calls for blending factorial and transplant experiments across manipulated or natural environmental gradients [19, 20, 29, 35].

\section{Stressors, stoichiometry and community and ecosystem function}

Evidence is beginning to show that predator presence in ecosystems can be an important drivers of whole ecosystem stoichiometry by instigating trait-mediated indirect effects that determine everything from the distribution of elements among trophic levels to the chemical content of 
organic matter that becomes decomposed by microbes [39,44]. These effects can be caused by direct effects such as predator-induced stress responses of prey [39], or from predation selecting on prey phenotype (body size and life-history strategy) [44], that in turn causes prey phenotypic state-dependence in physiology and hence nutrient demand. The important research challenge going forward is to resolve the relative importance of prey state due to stress responses imposed by predator presence (non-consumptive effects) from prey state imposed by predator selection (consumptive effects). This is especially important for scaling up the trade-off implications to whole ecosystem functioning. Theoretical analyses show that consumptive and non-consumptive effects can have decidedly different consequences for nutrient flux and pool size of nutrients among trophic levels [45*]. Indeed, predator non-consumptive effects driving prey phenotypic responses could lead to quantitatively stronger impacts because they alter prey production and efficiency rates, and recycling fluxes [45]: predictions that are consistent with experimental evidence [6].

A multiple-stressor framework can build on insight about prey phenotypic responses to predation stress to predict whole ecosystem nutrient cycling and stoichiometric balance [35, 37].

Generalizations are not yet possible because it remains uncertain whether any given stressor enhances or constrains organismal phenotypic capacity to respond to other stressors. This ultimately depends upon understanding whether multiple stressors synergistically or antagonistically perturb the same physiological pathways in individuals. Clearly the processes giving rise to within and between population organismal state ultimately determine whether and how organisms will respond. As such, knowing the cause of organismal state (degree of adaptation and plasticity) is critical because it ultimately shapes organismal trade-off decisions and thereby the nature of their trophic interactions in different environmental contexts. Thus, examining ecosystem functioning using the lens of organismal state-dependent effects on ecological stoichiometry offers considerable promise for operationally linking predation riskforaging trade-off behavior with eco-evolutionary processes.

\section{Conclusions}

Clearly, we are at a new frontier in efforts to link behavioral ecology with community and ecosystem functioning. The available empirical examples, although few in number, make it clear that our understanding of the connections between individual behavior and community and ecosystem processes might be advanced by examining how evolutionary and ecological context shape organismal state and how that scales to whole ecosystem processes. Pushing the bounds of this frontier, by developing new theory, and testing new and existing theory using new factorial, transplant experimental approaches is especially key to this endeavor. Without such a new approach, the shifting adaptive landscapes due to ongoing global change in multiple environmental variables will challenge the ability to predict organismal, population and ecosystem responses to multiple environmental stressors [17, 37].

\section{Acknowledgments}

We thank Burt Kotler for helpful comments. This work was supported by NSF grant DEB1354762 to OJS and NSF OCE-0963010 to GCT.

\section{References and recommended reading}


Papers published within the period of review, have been highlighted as:

${ }^{*}$ of special interest

1. Sih A: Optimal behavior: can foragers balance two conflicting demands? Science 1980, 210:1041-1043.

2. Abrams PA: Foraging time optimization and interactions in food webs. Am Nat 1984, 124:80-96.

3. Werner EE, Peacor SD: A review of trait-mediated indirect interactions in ecological communities. Ecology 2003, 84: 1083-1100.

4. Preisser, E, Bolnick DI, Benard MF. Scared to death? The effects of intimidation and consumption in predator-prey interactions. Ecology 2005, 86: 501-509.

5. Schmitz OJ: Resolving Ecosystem Complexity. Princeton: Princeton University Press; 2010.

6. Trussell GC, Schmitz OJ: Species functional traits, trophic control, and the ecosystem consequences of adaptive foraging in the middle of food chains In Trait-Mediated Indirect Interactions: Ecological and Evolutionary Perspectives. Edited by Ohgushi T, Schmitz OJ. Holt RD. Cambridge: Cambridge University Press; 2012.

7. Schmitz OJ, Buchkowski RW, Burghardt KT, Donihue CM. Functional traits and trait-

* mediated interactions: connecting community-level interactions with ecosystem functioning. Adv Ecol Res 2015, 52:319-344.

A conceptual advance on how to relate variation in expression of functional traits of microbes, plants, herbivores and detritivores in different environment contexts to trait-mediated indirect effects in ecosystems.

8. Loehle, C. Pechman HK. Evolution: the missing link in systems ecology. Am Nat 1988, 132:884-899.

9. Holt RD. 1995. Linking species and ecosystems: where is Darwin? In Linking Species and Ecosystems. Edited by Jones C, Lawton JH. London: Chapman and Hall, 1995.

10. Petorelli N, Hillborn A, Duncan C, Durant SM. Individual variability: the missing

* component to our understanding of predator-prey interactions. Adv Ecol Res 2015, 52:19-44.

A review of how variation in predator and prey populations leads to variation in prey selection by predator species and variation in prey species responses to predation risk.

11. Clark, CW, Mangel M. Dynamic Modeling in Behavioral Ecology. Princeton Univ. Press, 2000.

12. Kotler, BP, Brown, JS, Bouskila, A. Apprehension and time allocation in gerbils: the effects of predatory risk and energetic state. Ecology 2004, 85:917-922.

13. Ovadia O. Schmitz OJ. Linking individuals with ecosystems: experimentally identifying the relevant organizational scale for predicting trophic abundances. Proc Nat Acad Sci USA 2002, 99: 12927-12931. 
14. Matassa CM, Trussell GC. Prey state shapes the effects of temporal variation in * predation risk. Proc $R$ Soc B 2015, 281: 20141952.

An experimental demonstration of how prey foraging and life-cycle developmental responds to temporal variation in predation risk mediated by prey energetic state.

15. Trussell GC, Matassa CM, Luttbeg $B$. The effects of variable predation risk on foraging and growth: less risk is not necessarily better. Ecology 2011, 92: 1799-1806.

16. Sheriff MJ, Thaler JS. Ecophysiological effects of predation risk; an integration across disciplines. Oecologia 2014, 176:6-7-611.

17. Todgham AE, Stillman JH. Physiological responses to shifts in multiple environmental stressors: relevance to a changing world. Integr Comp Biol 2013, 53:539-544.

18. McNamara JM, Buchanan K.L. Stress, resource allocation and mortality. Behav Ecol 2005, 16:1008-1017.

19. Matassa CM, Trussell GC. Effects of predation risk across a latitudinal temperature * gradient. Oecologia 2015, 177:775-784.

Experimental demonstration of the importance of considering phenotypic adaptations and responses of populations across a geographic range to develop general understanding of variation in trait-mediated indirect effects in the face of thermal stress.

20. Ruehl CB, Trexler JC. Reciporocal transplant reveals trade-off of resource quality and * predation risk in the field. Oecologia 2015, 179:117-127.

Reveals the importance of using transplant experiments to disentangle the interplay of multiple environmental stressors on the strength of trait-mediated indirect interactions involving different populations of a prey species.

21. Matassa CM, Donelan SC, Luttbeg B, Trussell GC. Resource levels and prey state influence antipredator behavior and strength of nonconsumptive predator effects. Oikos 2016, DOI: 10.1111/oik.03165.

22. Gravem SA, Morgan SG. Prey state alters trait-mediated indirect interactions in rocky

* tidepools. Funct Ecol 2016, DOI: 10.1111/1365-2435.12628.

Experimental demonstration of how prey state influences spatial variation the strength of traitmediated indirect interactions to create spatial mosaics of top-down control strength.

23. Clark CW. Antipredator behavior and the asset-protection principle. Behav Ecol 1994, 5:159-170.

24. Ferrari, MCO, Sih, A, Chivers DP. The paradox of risk allocation: a review and prospectus. Anim Behav 2009, 78: 579-585.

25. Conover DO, Schlutz ET. Phenotypic similarity and the evolutionary significance of countergradient variation. Trends Ecol Evol 1995, 10:248-252.

26. Freidenburg, KL, Skelly DK. Microgeographic variation in thermal preference by an amphibian. Ecol Lett 2004, 7:369-373. 
27. Schoener TW. The newest synthesis: understanding the interplay of evolutionary and ecological dynamics. Science 2011, 331:426-429.

28. Ghalambor CK, McKay JK, Carroll SP, Reznick DN. Adaptive versus non-adaptive phenotypic plasticity and the potential for contemporary adaptation in new environments. Funct Ecol 2007, 21:394-407.

29. Hendry AP. Key questions on the role of phenotypic plasticity in eco-evolutionary

* dynamics. J Hered 2015, DOI: 10.1093/jhered/esv060.

A conceptual overview on the need to consider population-level phenotypic plasticity and local adaptation as a continuum to advance understanding of eco-evolutionary dynamics of ecological communities.

30. Relyea RA, Edwards K. What doesn't kill you makes you sluggish: how sublethal pesticides alter predator-prey interactions. Copeia 2010, 2010:558-567.

31. Stoks R, Swillen I, De Block M. Behaviour and physiology shape the growth accelerations associated with predation risk, high temperatures and southern latitudes in Ischnura damselfly larvae. J Anim Ecol, 2012, 81, 1034-1040.

32. Culler LE, McPeek MA, Ayers MP. Predation risk shapes thermal physiology of a

* predaceous damselfly. Oecologia, 2014, 176, 653-660.

An experimental demonstration of how predation risk and temperature interact as multiple stressors on prey performance.

33. Seebacher F, Grigalchik VS. Developmental thermal plasticity of prey modifies the * impact of predation. J Exp Biol 2015, 218, 1402-1409.

An experimental demonstration of how predation risk and temperature interact as multiple stressors to determine path dependency in prey development that causes state-dependent effects on prey stoichiometry.

34. Van Dievel M, Janssens L, Stoks R. Short- and long-term behavioural, physiological

* and stoichiometric responses to predation risk indicate chronic stress and compensatory mechanisms. Oecologia 2015, DOI 10.1007/s00442-015-3440-1.

An experimental demonstration of how predation risk and temperature interact as multiple stressors to influence prey metabolism and elemental stoichiometry.

35. Schmitz OJ. Global climate change and the evolutionary ecology of ecosystem function. Ann NY Acad Sci 2013, 1297:61-72.

36. Miller LP, Matassa CM, Trussell GC. Climate change enhances the negative effects of predation risk on an intermediate consumer. Glob Change Biol 2014, 20, 3834-3844.

37. Rosenblatt $A E$, Schmitz OJ. Interactive effects of multiple climate change variables on trophic interactions: a meta-analysis. Climate Change Responses 2014, 1:8

38. McPeek MA, Grace M. Richardson, J.M L. Physiological and behavioral responses to predators shape the growth/predation risk trade-off in damselflies. Ecology 2001, 82:1535-1545. 
39. Hawlena D, Schmitz, OJ. Herbivore physiological response to fear of predation and implications for ecosystem nutrient dynamics. Proc Nat Acad Sci USA 2010, 107: 15503-15507.

40. Thaler JS, McArt SH, Kaplan I. Compensatory mechanisms for ameliorating the fundamental trade-off between predator avoidance and foraging. Proc Nat Acad Sci USA 2012, 109:12075-12080.

41. Costello DM, Michel MJ. Predator induced defenses in tadpoles confound body stoichiometry predictions of the general stress paradigm. Ecology 2013, 94:2229-2236.

42. Dalton CM, Flecker AS. Metabolic stoichiometry and the ecology of fear in Trinidadian guppies: consequences for life-histories and stream ecosystems. Oceologia, 2014, 176:691-701.

43. Guariento RD, Carniero LS, Jorge JS, Borges AN, Esteves FA, Caliman A. Interactive effects of predation risk and conspecific density on the nutrient stoichiometry of prey. Ecol Evol 2015, 5:4747-4756.

44. El -Sabaawi RW, Marshall MC, Bassar RW, López-Sepulchre A, Plakovacs, EP, Dalton C. Assessing the effects of guppy life history evolution on nutrient cycling: from experiments to the field. Freshwater Biol 2015, 60:590-601.

45. Leroux SJ, Schmitz OJ. Predator-driven elemental cycling: the predation and risk * effects on ecosystem elemental cycling. Ecol Evol 2015, 5:4976-4988.

Theory that advances understanding of the single and combined effects of predation and stress from predation risk on whole ecosystem stoichiometry. 


\section{Figure 1.}

Developing a modern approach to studying trait-mediated indirect interactions in communities and ecosystems requires moving beyond a classic view $(\mathbf{A})$ that considers species typologically - that is, all individuals within a population are viewed as responding alike to predators and resource availability-according to the typical mean population trait value. Such a typological perspective holds that all prey species (blue circles) choose different resource species (green circles) and respond to stressors like predators (magenta circles) in the same way. A modern approach (B) recognizes that species are ensembles of individuals that vary in their traits, which causes withinpopulation state dependence. In such a view, different sized prey (different sized blue circles) will respond differently to their predators and choose a different spectrum of resources. This state-dependence leads to different magnitudes of trait-mediated indirect effect strengths and the species composition of the resource base. The exact nature of the state-dependent response may further depend on the evolutionary history of geographically separated local populations. Evolutionary history (C) may create another form of state dependence-between population state-dependence-because it leads to different constraints on the ability of populations to respond to environmental change and multiple stressors (ranging from adaptive plasticity to local adaptation produced by, for example, genetic polymorphism or genetic canalization). This means that modern approaches to understanding context-dependency in trait-mediated indirect effects need to conduct factorial transplant experiments across geographic locations to identify the potential causes and consequences of population state-dependence. Such experiments reveal the degree to which individuals in different populations with similar trait magnitudes (e.g. body size) are adaptively plastic (dashed red arrow) or locally adapted due to genetic canalization (solid red arrow) in the way they trade-off predationrisk with resource selection. This insight advances the ability to predict the community and ecosystem level consequences of global environmental change. 
(C) Among-Population

State Dependence

(B) Within-Population State Dependence
Phenotypic plasticity

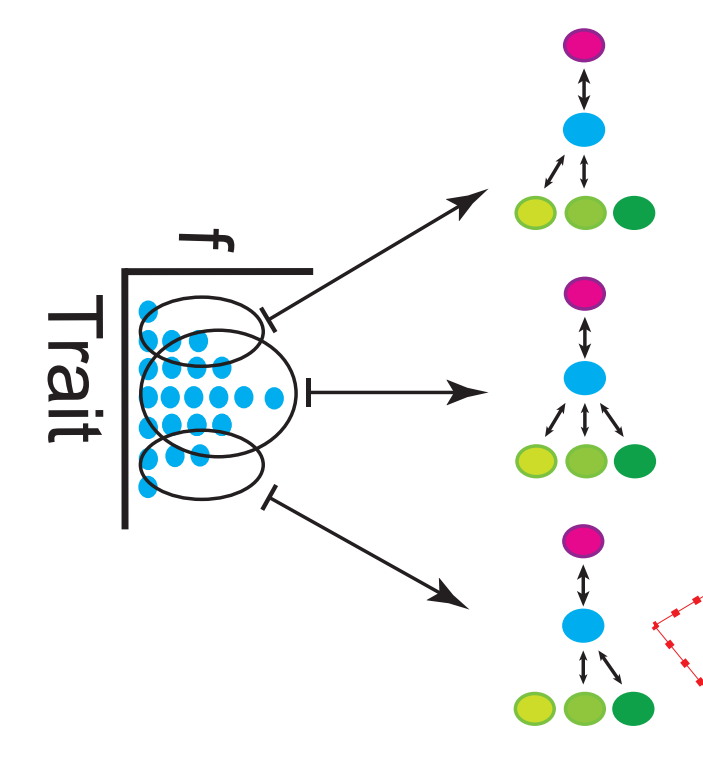

Typology
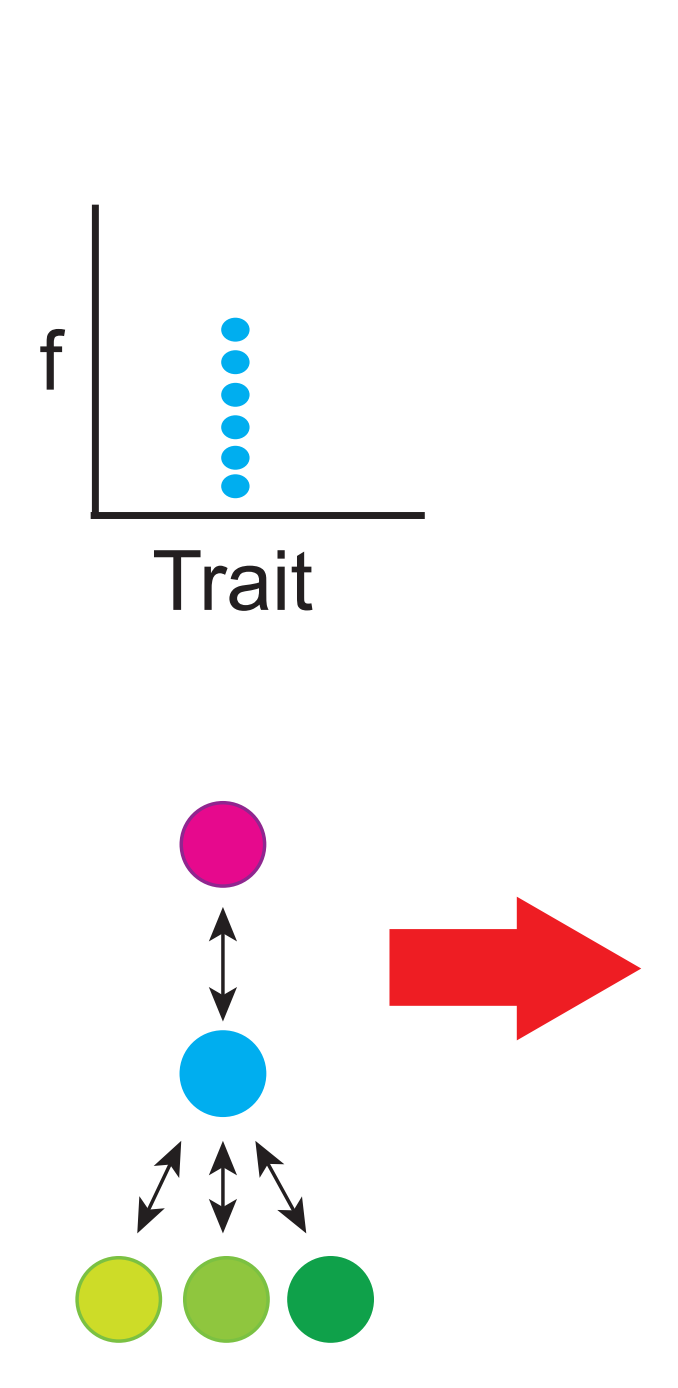

(A) Within-Population



Local adaptation

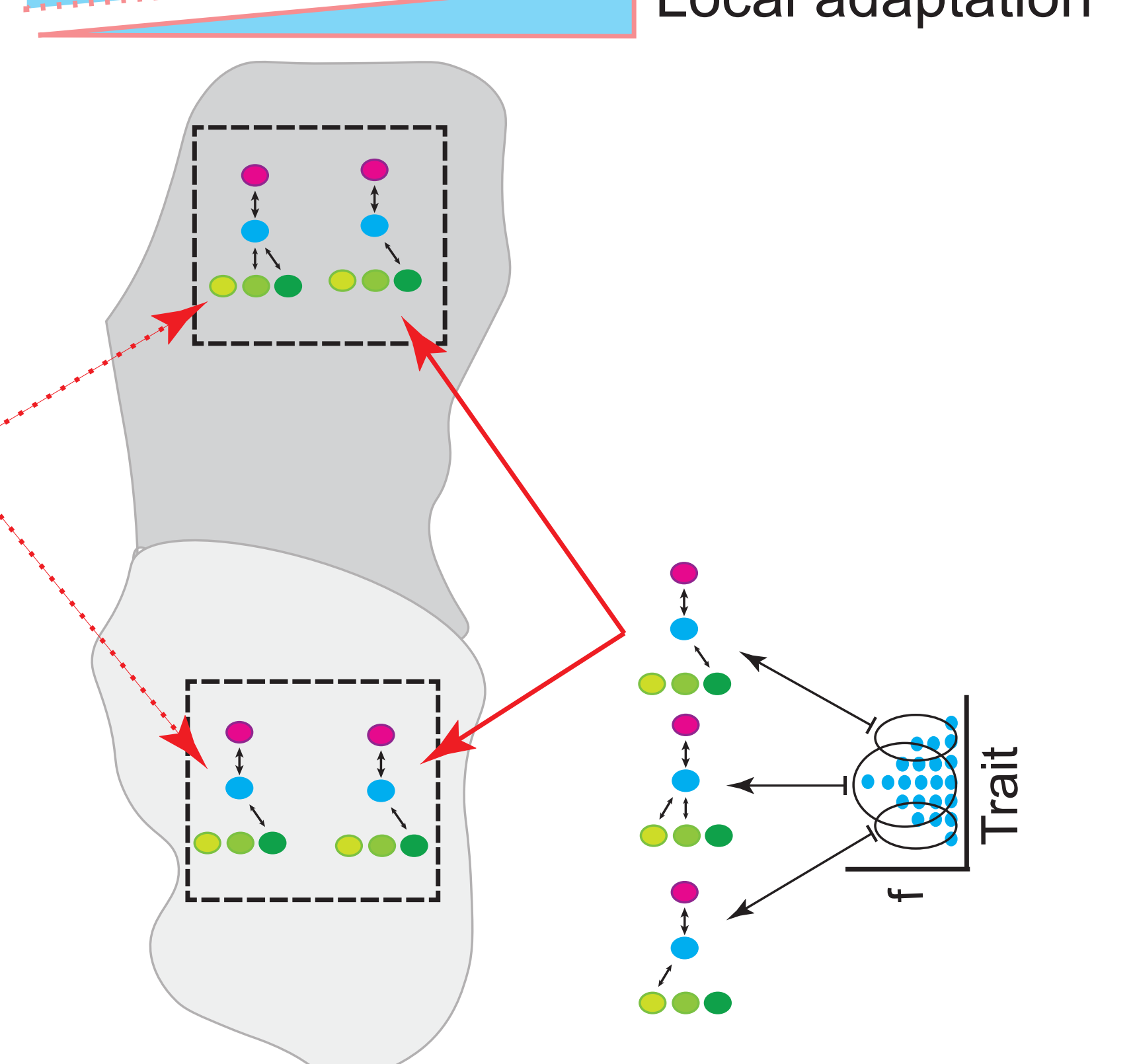




\section{Graphical Abstract}

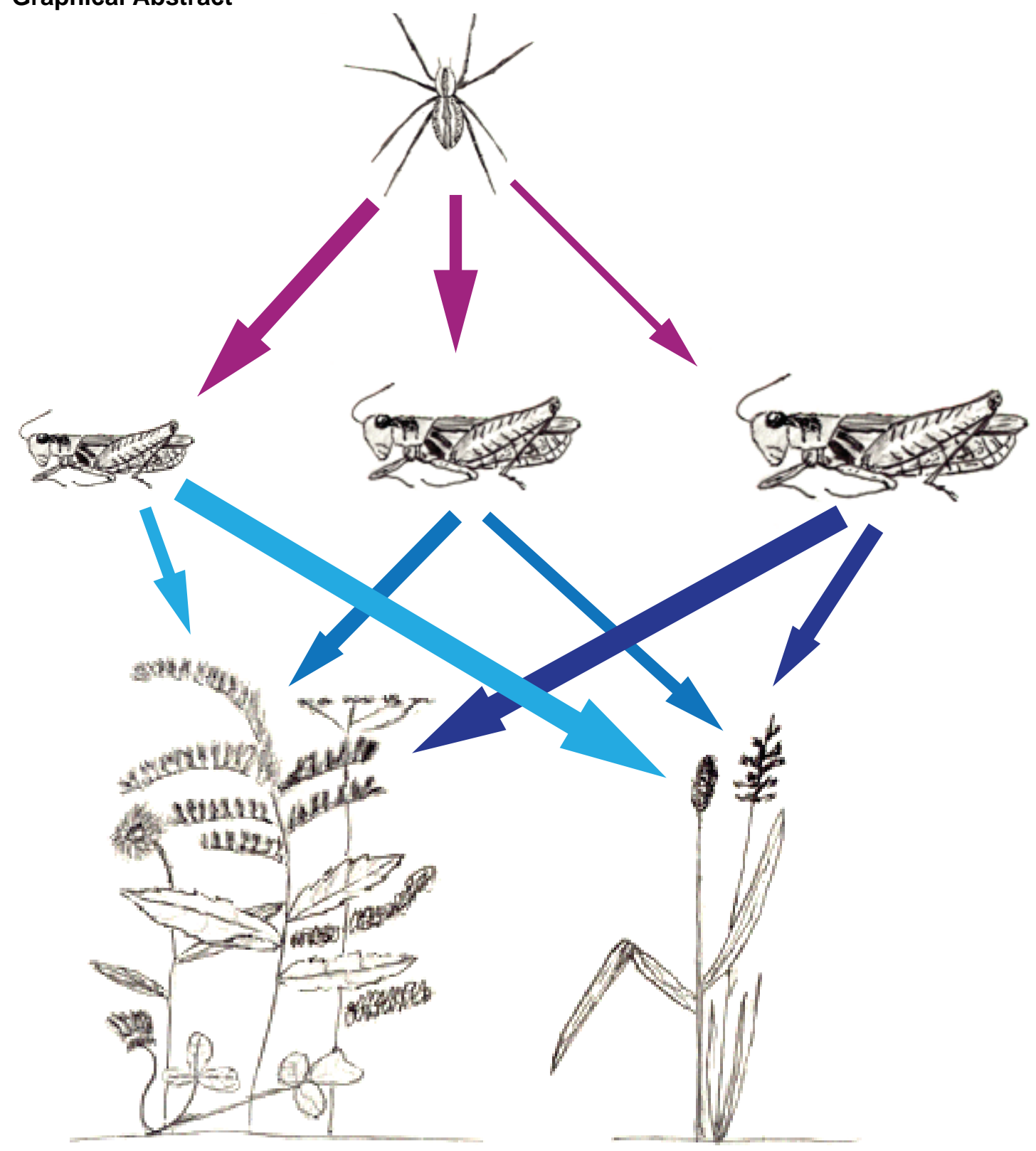

sonksy

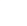

